\title{
Microstructure, Microhardness and Corrosion Resistance of WE43 Alloy Based Composites after High-Pressure Torsion
}

\author{
Petr Straumal ${ }^{1,2}$ (), Natalia Martynenko ${ }^{1,2}$, Askar Kilmametov ${ }^{3, *}$, Aleksey Nekrasov ${ }^{4}$ and \\ Brigitte Baretzky ${ }^{3}$ \\ 1 A.A. Baikov Institute of Metallurgy and Materials Science RAS, 119334 Moscow, Russia; \\ straumal.peter@yandex.ru (P.S.); nataliasmartynenko@gmail.com (N.M.) \\ 2 Institute of new materials and nanotechnologies, National University of Science and Technology «MISiS», \\ 119049 Moscow, Russia \\ 3 Institute of Nanotechnology, Karlsruhe Institute of Technology, 76344 Eggenstein-Leopoldshafen, Germany; \\ brigitte.baretzky@kit.edu \\ 4 Institute of Experimental Mineralogy, RAS, 142432 Chernogolovka, Russia; alexei.nekrasov@iem.ac.ru \\ * Correspondence: askar.kilmametov@kit.edu
}

Received: 30 July 2019; Accepted: 12 September 2019; Published: 15 September 2019

\begin{abstract}
The structure and properties of a composite consisting of Mg-Y-Nd-Zr alloy (WE43) and various oxides are studied. The particles of the WE43 powder were coated by the nanocrystalline oxide layer by means of a wet chemical deposition process. After that the powder is compressed into solid samples and deformed using high-pressure torsion at room temperature. A second phase is present, both, in pure WE43 alloy and in the one with deposited oxides. We observed that the modification of the alloy by the oxide layer deposition and deformation by high-pressure torsion changes the phase composition and properties of the samples. The samples modified by $\mathrm{TiO}_{2}$ showed the best microhardness and corrosion resistance.
\end{abstract}

Keywords: nanocrystalline metals; magnesium alloys; nanocrystalline oxides; severe plastic deformation; high-pressure torsion

\section{Introduction}

The properties of nanocrystalline metallic materials, such as mechanical characteristics, corrosion rate, and others, came to the fore in the last few decades. Nanocrystalline materials showed unusual functional properties because they contain an extremely high proportion of atoms located at the grain boundaries and other defects in the crystal structure. The WE43 alloy containing rare earth metals (REM) is one of the most popular medical magnesium alloys [1]. Alloying with REM improves the corrosion resistance of magnesium [2] and also increases its strength. Another method of improving the strength characteristics is severe plastic deformation (SPD), which leads to the formation of ultrafine-grained structure in magnesium and its alloys [3]. This structure provides significant hardening [3-6] and also leads to an increase in the corrosion resistance and a decrease in gas evolution.

Among all the methods of obtaining nanocrystalline alloys, high-pressure torsion (HPT), which belongs to the family of methods of severe plastic deformation occupies a special place because it demonstrates a number of advantages in comparison with its competitors, for example, it makes it possible to produce nanocrystalline materials in relatively large volumes from both pure metals and alloys.

The goal of this work is to investigate the possibility of altering the strength and corrosion properties of the WE43 alloy (a commercially available bioresorbable magnesium alloy 
Mg-3.56\%Y-2.20\%Nd-0.47\%Zr, wt.\% and traces of other rare-earth elements) by introducing oxide particles of various metals into the alloy and processing it by SPD. For this, the phase composition and properties of severely plastically deformed metal-ceramic composites based on the magnesium alloy WE43 modified with aluminum, titanium, magnesium and zinc oxides were studied.

Usually, the Mg-based composites can be fabricated by the casting or ball-milling process. The casting methods can be used to fabricate large parts at a low cost but the main problem is the occurrence of coarse microstructural features and comparatively large grain sizes in the final samples, resulting in low strength. Moreover, the reactions between the $\mathrm{Mg}$ matrix and the reinforcements cause the degradation of the composites. Another difficulty arising in the production of $\mathrm{Mg}$ composites by casting methods is that it is complicated to introduce fine reinforcement particles into the melt, especially when the volume fraction of the reinforcement is large. Usually, large ceramic particles with a size of a few micrometers are added. The process is more complicated when the particle size is much smaller than a tenth of a micrometer or when it is of nanometer size. Therefore, ball-billing is often chosen as the preparation method for Mg-alloys and second component composites.

Numerous Mg-oxide composites were produced by the ball-milling technique. Zhenglong et al. produced a nanocrystalline composite of $\mathrm{Mg}-3 \mathrm{Ni}-2 \mathrm{NiO}$ (wt.\%) by mechanical milling with nickel nano-powders under the hydrogen pressure [7]. Song et al. prepared a milled melt-spun Mg-23.5 wt. $\% \mathrm{Ni}$ with $10 \%$ nanocrystalline $\mathrm{Nb}_{2} \mathrm{O}_{5}$ [8]. Li et al. mechanical milled $\mathrm{Mg}-20 \mathrm{wt} . \%$ $\mathrm{Ni}-1 \mathrm{wt} . \% \mathrm{TiO}_{2}$ under a hydrogen atmosphere which directly led to the composite $\mathrm{MgH}_{2}$, and $\mathrm{Mg}_{2} \mathrm{NiH}_{4}$ [9].

In this paper, the method of pyrolysis of organometallic compounds is used to deposit the oxide layer on the magnesium alloy powder. The idea is to crush the $\mathrm{Mg}$ alloy, cover the powder particles by a nanometer thick oxide layer, after that produce an Mg-ceramic composite by SPD. During the SPD process, the nanometer-thick oxide layer should break and mix with the Mg matrix, producing fine and well-distributed oxide particles. Earlier, we already successfully produced WE43 composites with $\mathrm{Al}_{2} \mathrm{O}_{3}$ and $\mathrm{ZnO}$ [10], now we are investigating the composites with a different proportion of $\mathrm{Al}_{2} \mathrm{O}_{3}$ and $\mathrm{ZnO}$ and, additionally, with $\mathrm{TiO}_{2}$ and $\mathrm{MgO}$.

\section{Experimental}

First, the WE43 alloy was mechanically crushed into particles of $0.5-1 \mathrm{~mm}$ size. Second, thin films of nanocrystalline $\mathrm{Al}_{2} \mathrm{O}_{3}, \mathrm{ZnO}, \mathrm{MgO}$, and $\mathrm{TiO}_{2}$ were deposited onto $\mathrm{WE} 43$ particles using pyrolysis of organometallic compounds. This method has been developed under the supervision of Prof. A.A. Myatiev (NUST "MISiS", Moscow, Russia) for the deposition of nanocrystalline singleand multi-component oxide films. By pyrolytic decomposition of organic precursors containing salts of metals and carbon acids, we produce nanocrystalline oxide films [11-15]. Carboxylates of metals formed by metal cations and anions of higher isomeric carboxylic acids containing $\alpha, \alpha$ branched acid residues are used as a precursor. Carboxylates are applied to the surface of the substrate as a solution in a mixture of the same carboxylic acids, the anions of which are part of the carboxylates.

Extraction methods are used to prepare such solutions. As the extractant, a mixture of higher isomeric carboxylic acids (HICA) was used, the general formula of which:<smiles>[R]C(C)(C)C(=O)O</smiles>

The hydrocarbon radical $\mathrm{R}$ can contain from one (pivalic acid) up to 12 carbon atoms.

Using this approach, the films can be deposited on various substrates. The deposited films are dense, non-porous, nanostructured, uniform, and non-textured. The chemical components in multicomponent films are distributed uniformly [16,17]. 
In order to deposit the oxides, the obtained powder was mixed with the organometallic solutions of aluminum or zinc and the resulting mixture was annealed in air at $400^{\circ} \mathrm{C}$. Thermal decomposition of the organic component of the solution and precipitation of films consisting of a metal oxide, the cation of which was introduced into the solution, occurs on the surface of the WE43 powder particles.

In the previous study [10], all of the later deformed WE43 powder was coated by an oxide layer. In this study, one part of the oxide-coated WE43 powder was mixed with two parts of uncoated WE43 powder. To mix these parts properly, they were mixed with heptane and put into an ultrasonic bath. After that, the mixture was completely dried on air.

After proper mixing of the coated and uncoated powders, the mixture was subjected to SPD by HPT. The powder was poured into the cavity in the lower anvil and compressed to obtain a solid pellet. Samples were deformed at a pressure of $6 \mathrm{GPa}$ for 5 revolutions with $1 \mathrm{rpm}$ rotation speed. The cavity in the lower anvil was of $0.2 \mathrm{~mm}$ depth.

The microhardness was measured using a 402 MVD Instron Wolpert Wilson Instruments tester (Aachen, Deutschland). The morphology of obtained samples and the composition of the matrix and second phase were studied by electron probe micro-analyzer (EPMA) using a Tescan Vega TS5130 MM scanning electron microscope (SEM, Brno, Czech) equipped with an Oxford Instruments energy dispersion spectrometer (Abingdon-on-Thames, UK).

The transmission electron microscopy (TEM) was performed on the FEI Titan 80-300 Transmission Electron Microscope (Thermo Fisher Scientific, Waltham, MA, USA) with a high-angle annular dark-field scanning transmission electron microscopy (HAADF-STEM) and EDAX S-UTW energy dispersive X-ray analysis (EDX) detectors.

The study of the corrosion properties was carried out using the method of measuring the volume of hydrogen released during corrosion [18]. As a test medium, a solution of $0.9 \% \mathrm{NaCl}$ in distilled water was used (physiological solution, $\mathrm{pH}=7$ ). Samples were prepared manually by mechanical grinding on abrasive paper (from P800 to P2500). The tests of hydrogen evolution were carried out at room temperature and at $37^{\circ} \mathrm{C}$. The test sample was placed in a solution and covered with a funnel and a burette filled with corrosive medium. The sample remained in a corrosive environment for a day, after which the volume of hydrogen released was measured. The corrosion rate $\left(\mathrm{mL} /\left(\mathrm{cm}^{2} *\right.\right.$ day)) for the method of hydrogen evolution was calculated by the Equation (1):

$$
\mathrm{R} \Delta \mathrm{v}=\mathrm{VH} / \mathrm{S} * \mathrm{~T}
$$

where $\mathrm{VH}$ is the volume of released hydrogen, $\mathrm{ml} ; \mathrm{S}$ is the surface area of the sample, $\mathrm{cm}^{2}$; $\mathrm{T}$-time, days.

\section{Results and Discussion}

Figure 1 shows the results of a study of the obtained samples by SEM with an EPMA analysis of the matrix composition and inclusions. Table 1 shows the measured compositions of inclusions corresponding to the numbered points in Figure 1. 

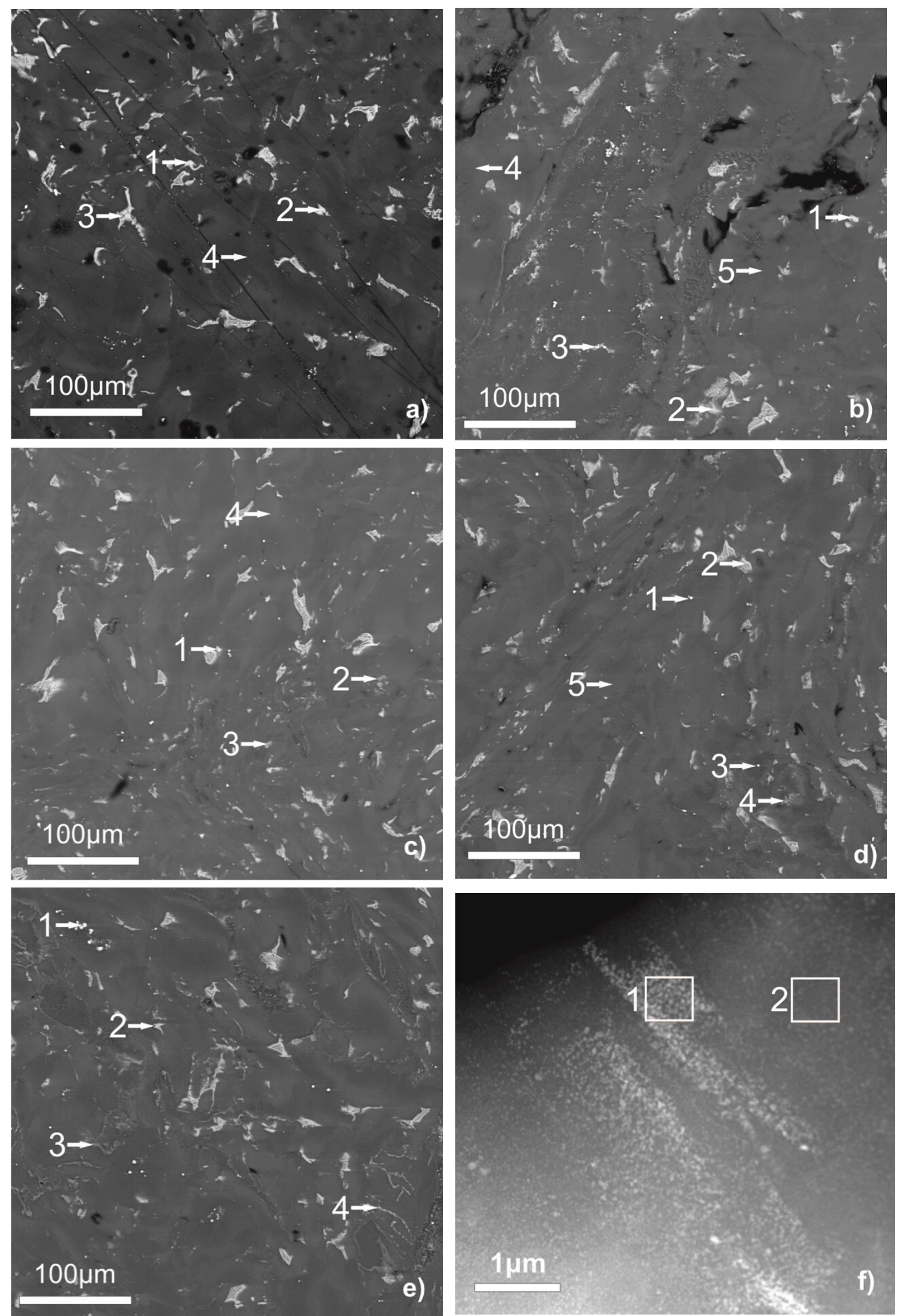

Figure 1. SEM micrographs of WE43 samples unmodified (a) [10], modified with aluminum oxide (b), titanium oxide (c), magnesium oxide (d), and zinc oxide (e) after deformation by high-pressure torsion. STEM micrograph of WE43 sample modified with aluminum oxide (f). 
Table 1. Results of the energy dispersive X-ray analysis (EDX) (conducted in SEM and TEM) of second phase precipitates in the samples of pure WE43 [10] and modified with aluminum, titanium, magnesium, and zinc oxide after high-pressure torsion (HPT) as shown in Figure 1. The composition is given in at.\%.

\begin{tabular}{|c|c|c|c|c|c|c|c|c|c|}
\hline \multirow{5}{*}{$\begin{array}{c}\text { WE43 6GPa5N } \\
\text { SEM }\end{array}$} & Point & $\mathrm{O}$ & $\mathrm{Mg}$ & $Y$ & $\mathrm{Zr}$ & La & $\mathbf{N d}$ & $\mathrm{Gd}$ & Dy \\
\hline & 1 & 3.2 & 86.38 & 4.4 & 0.02 & - & 5.99 & - & - \\
\hline & 2 & 3.28 & 87.1 & 4.16 & 0 & - & 5.45 & - & - \\
\hline & 3 & 2.33 & 85.89 & 5.32 & 0.03 & - & 6.44 & - & - \\
\hline & 4 & 6.83 & 91.99 & 0.95 & 0 & - & 0.22 & - & - \\
\hline \multirow{6}{*}{$\underset{\text { SEM }}{\mathrm{WE} 43+\underset{\mathrm{Al}_{2} \mathrm{O}_{3}}{6 \mathrm{GPa} 5 \mathrm{~N}}}$} & Point & O & $\mathrm{Mg}$ & $Y$ & Al & La & $\mathbf{N d}$ & Gd & Dy \\
\hline & 1 & 2.79 & 84.3 & 4.35 & 0.26 & 0.64 & 7.07 & 0.21 & 0.22 \\
\hline & 2 & 9.4 & 79.98 & 3.69 & 0.36 & 0.51 & 5.53 & 0.11 & 0.24 \\
\hline & 3 & 4.69 & 62.15 & 28.07 & 0.1 & 0.27 & 4.06 & 0.8 & 1.67 \\
\hline & 4 & 1.33 & 96.09 & 0.6 & 0.17 & 3.49 & - & - & 0.23 \\
\hline & 5 & 1.71 & 97.06 & 0.85 & 0 & 0 & 0.23 & 0.12 & 0.08 \\
\hline \multirow{5}{*}{ 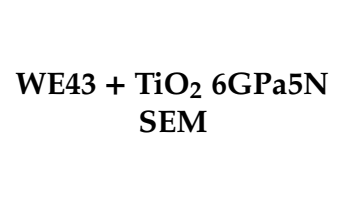 } & Point & O & $\mathrm{Mg}$ & $Y$ & $\mathbf{T i}$ & La & $\mathbf{N d}$ & $\mathrm{Zr}$ & Dy \\
\hline & 1 & 2.48 & 83.85 & 4.85 & 0 & 0.83 & 7.63 & 0.08 & 0.27 \\
\hline & 2 & 1.15 & 96.08 & 1.28 & 0.06 & 0.07 & 1.09 & 0.13 & 0.11 \\
\hline & 3 & 1.53 & 96.73 & 1.12 & 0 & 0.02 & 0.36 & 0.07 & 0.18 \\
\hline & 4 & 2.14 & 96.05 & 1.36 & 0 & 0 & 0.42 & 0.01 & 0.01 \\
\hline \multirow{6}{*}{$\underset{\text { SEM }}{\mathrm{WE} 43+\underset{\mathrm{MgO}}{\mathrm{MGPa} 5 \mathrm{~N}}}$} & Point & 0 & $\mathrm{Mg}$ & $Y$ & $\mathrm{Zr}$ & La & $\mathbf{N d}$ & $\mathrm{Gd}$ & Dy \\
\hline & 1 & 6.64 & 63.95 & 25.13 & 0.62 & 0.13 & 2.1 & 0.67 & 0.76 \\
\hline & 2 & 2.36 & 86.16 & 4.72 & 0.09 & 0.41 & 5.65 & 0.33 & 0.28 \\
\hline & 3 & 17.67 & 56.57 & 0 & 25.58 & 0.08 & 0.03 & 0.06 & 0 \\
\hline & 4 & 19.49 & 74.3 & 3.3 & 0.15 & 0.09 & 2.33 & 0.12 & 0.23 \\
\hline & 5 & 1.66 & 96.53 & 1.25 & 0.3 & 0 & 0.19 & 0 & 0.07 \\
\hline \multirow{5}{*}{ 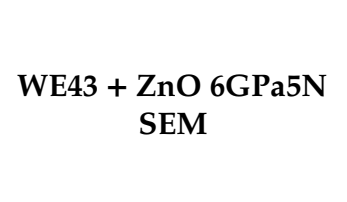 } & Point & O & $\mathrm{Mg}$ & $Y$ & $\mathrm{Zn}$ & $\mathrm{Zr}$ & $\mathrm{La}$ & $\mathrm{Nd}$ & Dy \\
\hline & 1 & 15.95 & 16.63 & 0 & 0.04 & 67 & 0.07 & 0.29 & 0 \\
\hline & 2 & 4.3 & 84.1 & 4.51 & 0 & 0.12 & 0.78 & 5.88 & 0.32 \\
\hline & 3 & 31.67 & 57.15 & 0.29 & 10.71 & 0.01 & 0 & 0.12 & 0.05 \\
\hline & 4 & 7.48 & 82.26 & 6.53 & 0.19 & 1.92 & 0.16 & 1.15 & 0.3 \\
\hline \multirow{3}{*}{ 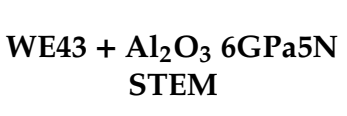 } & Area & O & $\mathrm{Mg}$ & $Y$ & Al & $\mathrm{Zr}$ & $\mathbf{N d}$ & C & - \\
\hline & 1 & 2.54 & 89.59 & 2.97 & 0.19 & 0.00 & 3.20 & 1.49 & - \\
\hline & 2 & 1.56 & 97.17 & 0.78 & 0.00 & 0.00 & 0.00 & 0.48 & - \\
\hline
\end{tabular}

The sample made only from the WE43 alloy, in the previous study [10], consisted of the Mg matrix with some $\mathrm{Y}$ addition and low Nd content and particles of the second phase, which hold a higher $\mathrm{Y}$ and Nd content.

As can be seen, the composition of a part of the second phase particles in the WE43 alloy after the HPT changes with the addition of oxides. The second phase particles in the pure WE43 alloy after HPT contain only the rare earth elements from the alloy. After modifying the WE43 alloy by oxides prior to HPT, a part of the second phase particles in the samples contains $\mathrm{Al}$, $\mathrm{Ti}$, or $\mathrm{Zn}$ from the oxide; It is observed as in SEM with a lower resolution, as in HAADF-STEM with a higher resolution. The uncertainty for the $\mathrm{Al}$ content in the TEM investigation is 0.05 at.\%. The second phase of the samples with the addition of magnesium oxide, sometimes, shows a higher oxygen, $\mathrm{Zr}$, or Nd content. Besides that, the samples with the addition of zinc oxide contain thin layers of the second phase on the borders of the initial particles or grain agglomerates, thus providing a higher strength.

Under the action of SPD, the mobility of atoms and the mass transfer rate are increased to the extent typical for diffusion during annealing at elevated temperatures. This leads to the mixture of the magnesium alloy WE43 with oxides added prior to the SPD.

Figure 2 shows the fine structure of the samples obtained by a dark-field TEM study. The DF TEM micrographs show that the sample made only of the WE43 alloy consists of the matrix with some second phase particles mostly in the form of bigger $(100-500 \mathrm{~nm})$ particles. The situation with the 
samples modified by the $\mathrm{Al}$ and $\mathrm{Zn}$ oxide is different. There the second phase is presented by bigger particles and highly dispersed small (down to $5 \mathrm{~nm}$ ) equiaxed particles. Figure $1 \mathrm{f}$ demonstrates the fine second phase particles, which are typical for the samples with oxides after SPD. During the SPD, the oxide layer on the surface of the powder particles breaks into fine oxide particles, which act as the segregation centers for the second phase. That promotes a more dispersive second phase segregation as we showed in our previous work [10].

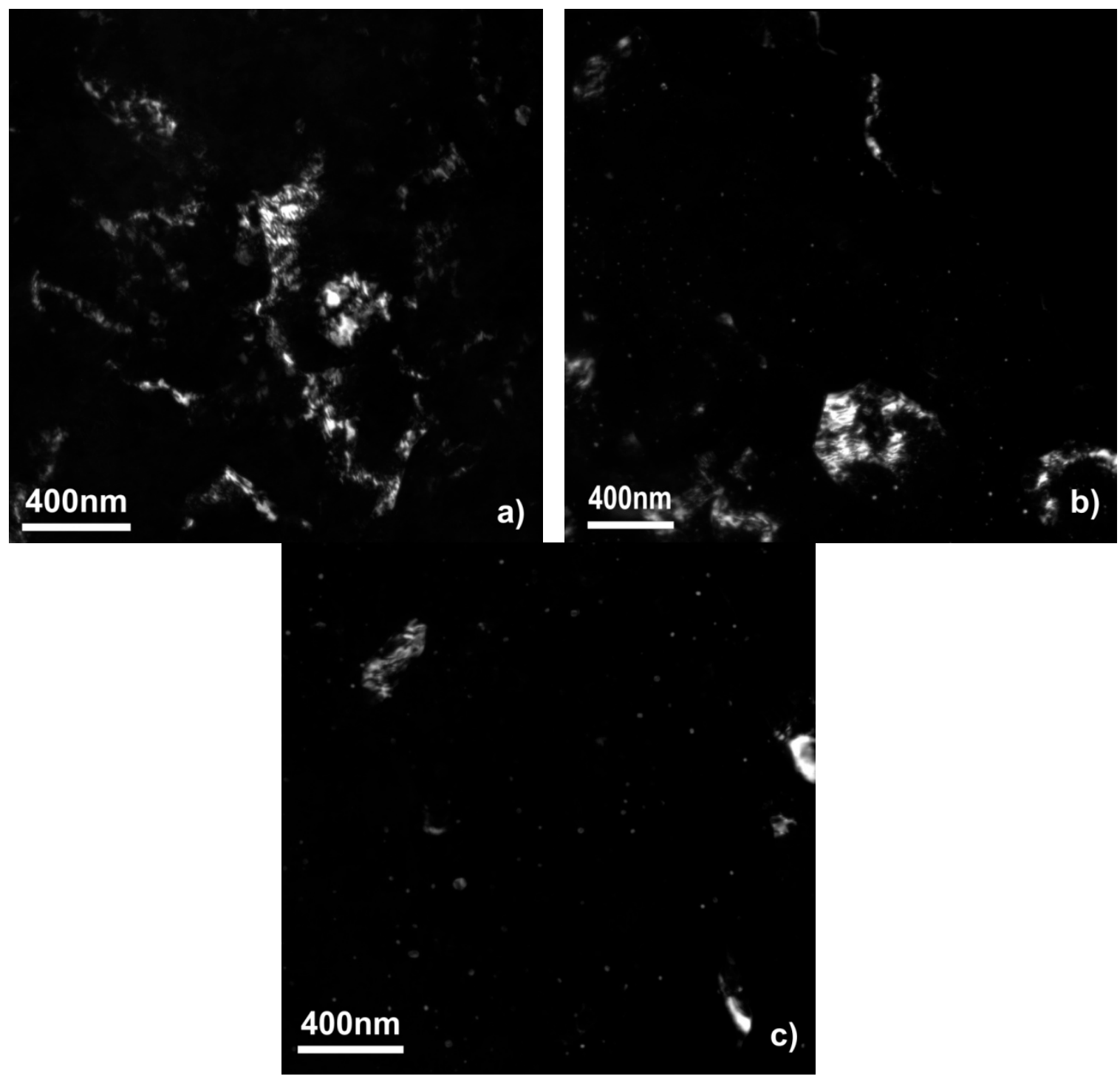

Figure 2. Dark-field (DF) TEM micrographs of WE43 samples unmodified (a), modified with aluminum oxide (b), and zinc oxide (c) after deformation by high-pressure torsion.

The microhardness of the samples after the HPT was measured as an array of $15 \times 15$ points over the whole area of the samples. The measured microhardness on the middle of the sample radius after HPT is, as follows: for WE43 with aluminum oxide-969 $\pm 34 \mathrm{MPa}$, for WE43 with titanium oxide-1,017 $\pm 36 \mathrm{MPa}$, for WE43 with magnesium oxide-997 $\pm 36 \mathrm{MPa}$, and for WE43 with zinc oxide-986 $\pm 32 \mathrm{MPa}$.

The microhardness measured in the previous study [10] for pure WE43 after crushing and HPT is $1299 \pm 17 \mathrm{MPa}$ and for WE43 with aluminum oxide is $551 \pm 14 \mathrm{MPa}$, for WE43 with zinc oxide is $867 \pm 21 \mathrm{MPa}$. The value for the pure deformed WE43 corresponds well with the microhardness of as-cast WE43 deformed by HPT [3]; this shows that the crushing of the alloy prior to the HPT did 
not affect the microhardness of the alloy. However, it should be noted that the addition of all oxides reduces the microhardness compared to the alloy deformed without oxides. Insufficient mixing of the oxides with the alloy powder particles due to their rather large size $(0.5-1 \mathrm{~mm})$ may be the most likely reason for this behavior. Therefore, the structure contains oxide regions and regions with a deformed alloy, which leads to a moderate decrease in the average microhardness. This is also probably the cause of why microhardness does not increase due to the pinning of dislocations by oxide particles. The use of more dispersed particles of alloy powder can be one possible way to solve this problem.

The corrosion tests result in Figure 3 show us the different corrosion rates of the pure WE43 after HPT and oxide modified WE43 samples after HPT. Clearly, all oxides lower the corrosion rate but the $\mathrm{Al}_{2} \mathrm{O}_{3}$ and $\mathrm{TiO}_{2}$ perform best in that task.

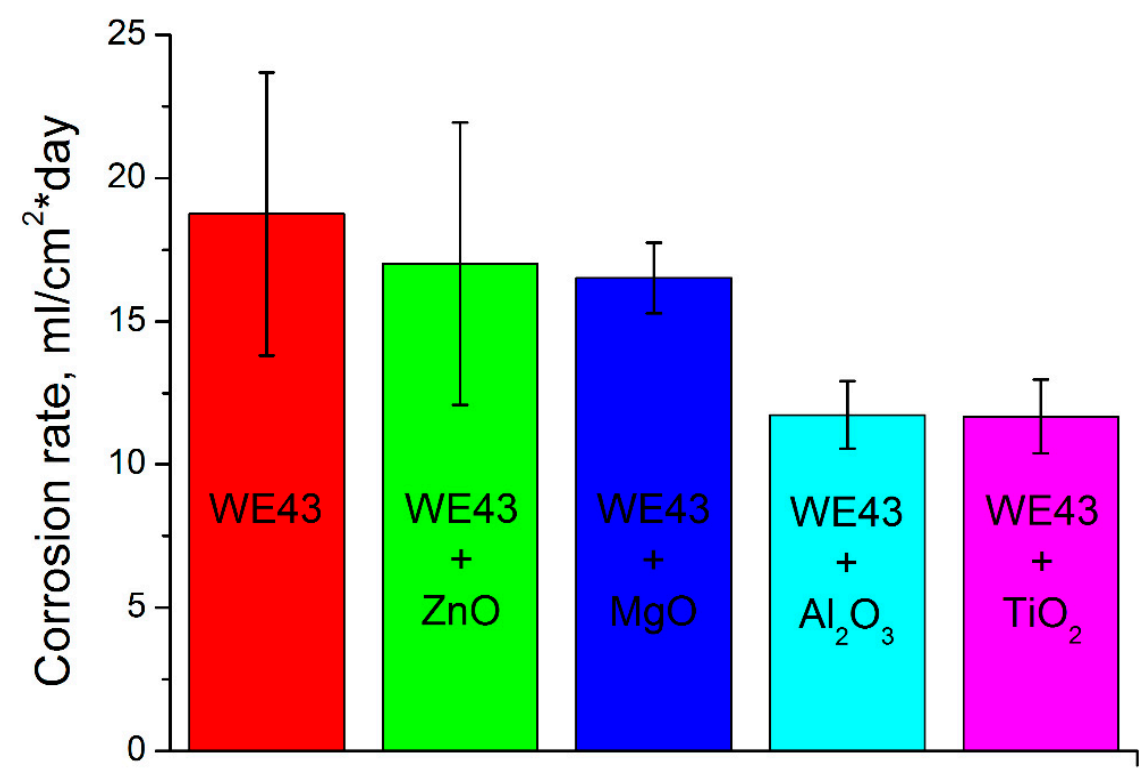

Figure 3. Results of pure and oxide-modified WE43 alloy corrosion tests.

\section{Conclusions}

Samples (magnesium alloy)/(oxide) composites were successfully produced. They are dense and it is possible to study their structure and properties.

The oxide metals enter the second phase particles and promote more dispersive second phase segregation. That changes the phase composition after the SPD process and, accordingly, alters the properties of the composite.

The strength of the samples increases compared to the results of the previous study; this is a result of a lower amount of modificating oxide. This reduction was planned and the results show that our study succeeded.

The corrosion resistance is higher for all modifying oxides compared to the pure WE43 after HPT but the best results were achieved for the $\mathrm{TiO}_{2}$. The same is true for the microhardness.

Author Contributions: Conceptualization, A.K., P.S. and N.M.; Methodology, P.S. and A.K.; Formal Analysis, P.S., N.M. and A.N.; Investigation, P.S., N.M. and A.N.; Resources, A.K.; Writing-Original Draft Preparation, A.K., P.S. and N.M.; Writing-Review \& Editing, A.K., P.S., N.M. and B.B.; Supervision, B.B.; Project Administration, B.B.; Funding Acquisition, P.S. and A.K.

Funding: This research was funded by the Russian Science Foundation under grant 17-72-10304 and performed in National University of Science and Technology «MISiS».

Acknowledgments: The work was funded by the Russian Science Foundation under grant 17-72-10304 and performed in National University of Science and Technology «MISiS». We acknowledge support by the KIT-Publication Fund of the Karlsruhe Institute of Technology (for publishing the work in an open access journal).

Conflicts of Interest: The authors declare no conflict of interest. 


\section{References}

1. Liu, Y.; Zheng, S.; Li, N.; Guo, H.; Zheng, Y.; Peng, J. Study on the in vitro degradation behavior of pure Mg and WE43 in human bile for 60 days for future usage in biliary. Mater. Lett. 2016, 179, 100-103. [CrossRef]

2. Li, N.; Guo, C.; Wu, Y.H.; Zheng, Y.F.; Ruan, L.Q. Comparative study on corrosion behaviour of pure Mg and WE43 alloy in static, stirring and flowing Hank's solution. Corros. Eng. Sci. Technol. 2012, 47, 346-351. [CrossRef]

3. Lukyanova, E.A.; Martynenko, N.S.; Shakhova, I.; Belyakov, A.N.; Rokhlin, L.L.; Dobatkin, S.V.; Estrin, Y.Z. Strengthening of age-hardenable WE43 magnesium alloy processed by high pressure torsion. Mater. Lett. 2016, 170, 5-9. [CrossRef]

4. Salandari-Rabori, A.; Zarei-Hanzaki, A.; Fatemi, S.M.; Ghambari, M.; Moghaddam, M. Microstructure and superior mechanical properties of a multi-axially forged WE magnesium alloy. J. Alloys Compd. 2017, 693, 406-413. [CrossRef]

5. Diez, M.; Kim, H.E.; Serebryany, V.; Dobatkin, S.; Estrin, Y. Improving the mechanical properties of pure magnesium by three-roll planetary milling. Mater. Sci. Eng. A 2014, 612, 287-292. [CrossRef]

6. Zhang, F.; Zhang, K.X.; Tan, C.W.; Yu, X.D.; Ma, H.L.; Wang, F.C.; Cai, H.N. Microstructure and mechanical properties of Mg-Gd-Y-Zr alloy processed by equal channel angular pressing. Trans. Nonferrous Met. Soc. China (English Ed.) 2011, 21, 2140-2146. [CrossRef]

7. Zhenglong, L.; Zuyan, L.; Yanbin, C. Cyclic hydrogen storage properties of Mg milled with nickel nano-powders and NiO. J. Alloys Compd. 2009, 470, 470-472. [CrossRef]

8. Song, M.Y.; Kwon, S.N.; Bae, J.S.; Hong, S.H. Hydrogen-storage properties of melt spun Mg-23.5 wt $\% \mathrm{Ni}$ milled with nano $\mathrm{Nb}_{2} \mathrm{O}_{5}$. J. Alloys Compd. 2009, 478, 501-506. [CrossRef]

9. Li, F.; Jiang, L.; Du, J.; Wang, S.; Liu, X.; Zhan, F. Investigations on synthesis and hydrogenation properties of Mg-20 wt $\% \mathrm{Ni}-1 \mathrm{wt} \% \mathrm{TiO}_{2}$ composite prepared by reactive mechanical alloying. J. Alloys Compd. 2008, 452, 421-424. [CrossRef]

10. Straumal, P.B.; Martynenko, N.; Amelina, D.; Nekrasov, A.; Dobatkin, S. Phase Composition and Properties of Magnesium-Ceramic Composites after High Pressure Torsion. Defect Diffus. Forum 2018, 385, $218-222$. [CrossRef]

11. Straumal, B.; Mazilkin, A.; Straumal, P.; Myatiev, A. Distribution of impurities and minor components in nanostructured conducting oxides. Int. J. Nanomanuf. 2008, 2, 253. [CrossRef]

12. Straumal, B.B.; Mazilkin, A.A.; Protasova, S.G.; Myatiev, A.A.; Straumal, P.B.; Baretzky, B. Increase of Co solubility with decreasing grain size in ZnO. Acta Mater. 2008, 56, 6246-6256. [CrossRef]

13. Straumal, B.; Baretzky, B.; Mazilkin, A.; Protasova, S.; Myatiev, A.; Straumal, P. Increase of Mn solubility with decreasing grain size in ZnO. J. Eur. Ceram. Soc. 2009, 29, 1963-1970. [CrossRef]

14. Straumal, B.B.; Mazilkin, A.A.; Protasova, S.G.; Myatiev, A.A.; Straumal, P.B.; Schütz, G.; van Aken, P.A.; Goering, E.; Baretzky, B. Magnetization study of nanograined pure and Mn-doped ZnO films: Formation of a ferromagnetic grain-boundary foam. Phys. Rev. B Condens. Matter Mater. Phys. 2009, 79, 1-6. [CrossRef]

15. Straumal, P.B.; Stakhanova, S.V.; Wilde, G.; Divinski, S.V. 44Ti self-diffusion in nanocrystalline thin $\mathrm{TiO}_{2}$ films produced by a low temperature wet chemical process. Scr. Mater. 2018, 149, 31-35. [CrossRef]

16. Straumal, B.B.; Myatiev, A.A.; Straumal, P.B.; Mazilkin, A.A.; Protasova, S.G.; Goering, E.; Baretzky, B. Grain boundary layers in nanocrystalline ferromagnetic zinc oxide. JETP Lett. 2010, 92, 396-400. [CrossRef]

17. Straumal, B.B.; Protasova, S.G.; Mazilkin, A.A.; Myatiev, A.A.; Straumal, P.B.; Schütz, G.; Goering, E.; Baretzky, B.; Schütz, G.; Goering, E.; et al. Ferromagnetic properties of the Mn-doped nanograined ZnO films. J. Appl. Phys. 2010, 108, 1-6. [CrossRef]

18. Song, G.; Atrens, A.; Suohn, D. An Hydrogen Evolution Method for the Estimation of the Corrosion Rate of Magnesium Alloys. Essent. Readings Magnes. Technol. 2014, 9781118858, 565-572. [CrossRef]

(C) 2019 by the authors. Licensee MDPI, Basel, Switzerland. This article is an open access article distributed under the terms and conditions of the Creative Commons Attribution (CC BY) license (http://creativecommons.org/licenses/by/4.0/). 